\title{
Relationship Between the Loudness Dependence of the Auditory Evoked Potential and the Severity of Suicidal Ideation in Patients with Major Depressive Disorder
}

\author{
Mingyu Hwang ${ }^{1}$, Yeon Jung Lee ${ }^{1}$, Minji Lee ${ }^{1}$, Byungjoo Kang ${ }^{1}$, Yun Sung Lee ${ }^{2}$, Jaeuk Hwang ${ }^{1}$, Sung-il Woo ${ }^{1}$, \\ Sang-Woo Hahn ${ }^{1}$ \\ ${ }^{1}$ Department of Psychiatry, Soonchunhyang University Seoul Hospital, Soonchunhyang University College of Medicine, Seoul, ${ }^{2}$ Department of \\ Medical Sciences, Graduate School of Soonchunhyang University, Asan, Korea
}

\begin{abstract}
Objective: The loudness dependence of the auditory evoked potential (LDAEP) is a reliable indicator that is inversely related to central serotonergic activity, and recent studies have suggested an association between LDAEP and suicidal ideation. This study investigated differences in LDAEP between patients with major depressive disorder and high suicidality and those with major depressive disorder and low suicidality compared to healthy controls.

Methods: This study included 67 participants: 23 patients with major depressive disorder with high suicidality (9 males, mean age $29.3 \pm 15.7$ years, total score of SSI-BECK $\geq 15$ ), 22 patients with major depressive disorder with low suicidality (9 males, mean age $42.2 \pm 14.4$ years, total score of SSI-BECK $\leq 14)$, and 22 healthy controls $(11$ males, mean age $31.6 \pm 8.7$ years). Participants completed the following assessments: Patient Health Questionnaire-9, Beck Depression Inventory-II, Beck Scale for Suicidal ideation, State Anxiety Scale of the State-Trait Anxiety Inventory, Beck Anxiety Inventory, and LDAEP (measured at electrode Cz).

Results: There were no sex-related differences among groups $(p=0.821)$. The high-suicidality group exhibited significantly higher LDAEP compared to the low-suicidality group $(0.82 \pm 0.79$ vs. $0.26 \pm 0.36, p=0.014)$. No significant differences were found between the control and high-suicidality $(p=0.281)$ or the control and low-suicidality groups $(p=0.236)$.

Conclusion: LDAEP was applied to demonstrate the association between serotonergic activity and suicidal ideation and suicide risk in major depression and may be a candidate of biological marker for preventing suicide in this study.
\end{abstract}

KEY WORDS: Depression; Suicidal ideation; Suicide risk; Biomarkers; Auditory evoked potential; Loudness dependence of auditory evoked potentials.

\section{INTRODUCTION}

South Korea has had the highest suicide rate among the OECD countries during most of the period from 2003 to the present; in 2018, the rate was 26.6 per 100,000 people [1]. In Korea, suicide is the second leading cause of death following cancer [2]. Moreover, the direct and indirect socioeconomic costs of suicide amount to 6.5 tril-

Received: July 9, 2020 / Revised: September 22, 2020

Accepted: September 29, 2020

Address for correspondence: Sang-Woo Hahn

Department of Psychiatry, Soonchunhyang University Seoul

Hospital, Soonchunhyang University College of Medicine, 59

Daesagwan-ro, Yongsan-gu, Seoul 04401, Korea

E-mail: ha5hn@schmc.ac.kr

ORCID: https://orcid.org/0000-0003-1662-5438 lion Korean won (KRW) per year, which is higher than that for cerebrovascular disease (5.1 trillion KRW), heart disease (5 trillion KRW), and diabetes (2.7 trillion KRW) [2]. Thus, suicide places a burden on individuals, families, and the nation. Because suicide is sudden, impulsive, difficult to predict [3], and brings about irreversible consequences, it is important to assess suicide risks in advance and prevent it when possible. Since the most common mental illness among suicide victims is depression [4-6], and suicidal ideation has been known to be a main predictor of suicide [7], it is necessary to evaluate the severity of suicidal ideation among depressed patients for early intervention to lower the suicide rate. Currently, common methods of evaluating suicidal ideation are

(c) This is an Open-Access article distributed under the terms of the Creative Commons Attribution Non-Commercial License (http://creativecommons.org/licenses/by-nc/4.0) which permits unrestricted non-commercial use, distribution, and reproduction in any medium, provided the original work is properly cited. 
self-report questionnaires and direct questions addressed to patients. However, it is difficult to accurately determine suicide risk when patients respond defensively. One study [8] suggested that a multi-faceted evaluation method including interviews about existing predispositions, stressors, symptoms, past history of suicidality, and protective factors be used for suicide prevention. However, this approach also has limitations due to the above reasons. Therefore, many clinicians have suggested the importance of including objective factors in suicide risk assessment, and efforts to find biological markers associated with suicide have been undertaken [9].

Patients with suicidal ideations exhibit a variety of biological abnormalities, such as in neurotransmitters and hormones [10-12]. Among such associations, the relationship between suicidal ideation and serotonin (5-HT) in the central nervous system has been studied the most. Serotonin is associated with emotional regulation or impulsivity [13]. The serotonin system is also connected to the frontal lobe, midbrain, and cerebellum, and serotonin is associated with major functions such as respiration, sleep, sexual desire, mood, and hormone secretion [14]. Serotonin deficiency may be associated with suicide, depression, and alcohol use disorders [15]. In relation to suicide, serotonin-related gene polymorphisms have been identified in depressed patients with suicidal ideation [16-18]. Serotonin may also affect suicide because it controls aggression and mood [19]. Various molecular modifications, and changes in serotonin levels and signaling, occur in individuals who are suicidal [20]. Positron emission tomography studies in individuals who attempted suicide have found a decrease in frontal lobe function, which is associated with serotonin [21]. To identify how serotonin acts as a neurotransmitter, it is necessary to measure central serotonin activity. Because serotonin cannot cross the blood-brain barrier [22], measurement of central serotonin activity has been performed using classic invasive methods, such as detecting serotonin metabolites in cerebrospinal fluid through spinal puncture or performing the Fenfluramine challenge test [23]. However, because these methods are clinically difficult to use, a simple and noninvasive method of measuring central serotonin activity is needed [24].

Loudness dependence of the auditory evoked potential (LDAEP) is regarded as effective and economic noninvasive indicator of central serotonergic function, and is thought to be inversely proportional to the central serotonergic activity in several studies [25-27]. Thus, when central serotonergic activity is high, the LDAEP level is reduced, and vice versa. LDAEP is assessed at the Cz electrode in EEG. Participants are given sound stimuli of 60 , $70,80,90$, and $100 \mathrm{~dB}$ intensity at random time intervals of $500-900 \mathrm{~ms}$, and LDAEP is measured by calculating the peak-to-peak N1/P2 amplitude as the slope of the linear regression. LDAEP has been reported to be a reliable indicator of central serotonin activity $[25,28]$. It is associated with emotional sensitivity, and LDAEP abnormalities have been reported in various psychiatric disorders involving serotonin, such as schizophrenia and mood disorders $[29,30]$. Previous studies on the relationship between suicide and LDAEP [24,31-33] have reported higher LDAEP in suicidal subjects compared to non-suicidal subjects. However, no studies on the use of the severity of suicidal ideation to identify high-risk suicide groups have been conducted. Not all patients with suicidal ideation attempt suicide, but the link has been reported in several studies [34,35]. Thus, assessing the severity of suicidal ideation is very important in determining the risk for suicide attempts [36]. Therefore, in this study, we examined LDAEP in a group with major depressive disorder and high suicidality, a group with major depressive disorder and low high suicidality, and a healthy control group to determine whether the groups differed in serotonergic activity according to the level of suicidal ideation.

\section{METHODS}

\section{Participants}

This cross-sectional study enrolled 46 patients who were diagnosed with major depressive disorder at the Soonchunhyang University Seoul Hospital and 25 healthy controls. Patients and controls aged 18-65 years were recruited from January 2019 to December 2019. The depression group was diagnosed using a structured clinical interview based on the criteria of the Diagnostic and Statistical Manual of Mental Disorders, 5th edition (DSM-5) [37]. Participants were requested to answer several questionnaires, and each participant was divided into high suicidal ideation (total score of SSI-BECK $\geq 15$ ) and low suicidal ideation group (total score of SSI-BECK $\leq 14$ ) by total SSI-BECK score. Patients who were unable to self-report due to head injury, neurological disorders, or severe 
medical diseases and those who did not agree to voluntary participation were excluded from the study. Normal hearing was confirmed in all participants using a $512 \mathrm{~Hz}$ tuning fork test [38]. Among the 71 participants, 4 (1 patient, 3 controls) who showed LDAEP waveforms that were difficult to analyze due to poor data were excluded. Consequently, a total of 67 participants (45 patients, 22 controls) were included. All participants, except for one, were right-handed. All participants completed a written agreement in accordance with the Declaration of Helsinki. This study was approved by the Institutional Review Board of Soonchunhyang University Hospital (No. 2019-04-003).

\section{Assessment}

\section{Demographic characteristics}

Participants were asked to provide their sex, age, educational background, and occupation. Education was classified into four categories: junior high school, with or without graduation (1); high school graduate (2); university graduate (3); and graduate degree (4).

\section{Beck Scale for Suicidal ideation (SSI-BECK)}

The SSI-BECK is a self-report questionnaire developed by Beck et al. [39] to measure the severity of suicidal ideation. The SSI-BECK is an important predictor of future suicidal behavior and is highly correlated with clinicians' assessments of suicide risk [40]. The SSI-BECK consists of 19 questions. Each item is rated on a 3-point Likert scale, with higher total scores indicating more severe suicidal ideation [39]. According to a study of the SSI-BECK by Shin et al. [40], scores of $0-8$ are considered normal, 911 mild suicidality, 12-14 moderate suicidality, and scores $\geq 15$ indicate severe suicidality. In this study, a total SSI-BECK score $\leq 14$ was taken to indicate low suicide risk (low-suicidality group) and $\geq 15$ was high suicide risk (high-suicidality group). The Korean version was standardized by Shin et al. [40] and well reflects the original questionnaire. The Cronbach's alpha was 0.81 [40].

\section{Patient Health Questionnaire-9 (PHQ-9)}

The PHQ-9 was developed by Kroenke et al. [41] as a tool to screen depression and assess its severity. The PHQ-9 comprises nine questions, each of which is evaluated on a 4-point Likert scale indicating how often symptoms have occurred in the past 2 weeks. Based on the total score, scores of 5-9 points are evaluated as slightly depressed, $10-14$ points as moderately depressed, 15-19 points as severely depressed, and $20-27$ points as an extremely severe depressed state [41]. The Korean version of PHQ-9 was translated by Han et al. [42] and the Cronbach's alpha was 0.81 [43].

\section{Beck Depression Inventory-II (BDI-II)}

The BDI-II is a self-report questionnaire consisting of 21 items that measure current depressive symptoms. It was developed by Beck et al. [44] as a supplementary correction of the BDI Scale based on criteria for the diagnosis of depressive disorder included in DSM-IV. Symptoms over the past 2 weeks are evaluated, and items such as sleep, appetite, worthlessness, avolition, irritability, and loss of concentration are included [45]. Based on the total score, scores of $0-9$ are interpreted as not depressed, $10-15$ as mildly depressed, 16-23 as moderately depressed, and 24-63 as severely depressed [44]. The Korean version of BDI-Il was translated by Sung et al. [45], and the Cronbach's alpha was over 0.80 [45].

\section{State Anxiety on the State-Trait Anxiety Inventory (SAI)}

The State - Trait Anxiety Inventory (STAI) is a self-report screening tool developed by Spielberger et al. [46,47] to measure state and trait anxiety. In general, trait anxiety refers to relatively stable differences among individuals in the intensity and frequency of past, present, and future anxiety tendencies, whereas state anxiety refers to feelings of subjective and consciously perceived tension and anxiety associated with heightened autonomic nervous system activity [46]. In this study, the Korean version of the SAI scale, which was standardized by Kim and Shin [48], was employed. The SAI uses a 4-point Likert scale for 20 questions asking about experiences of anxiety over a relatively short period, where $1=$ absolutely no, $2=$ no, 3 =yes, $4=$ definitely yes. The total score ranges from 20 to 80 points, and higher scores indicate greater state anxiety $[47,48]$. Based on the total score, state anxiety is considered slightly elevated with scores of $52-56$, significantly elevated at $57-61$, and extremely elevated at $\geq 62$ points [47]. For the Korean SAI, the Cronbach's alpha was 0.89 [48].

\section{Beck Anxiety Inventory (BAI)}

The $\mathrm{BAI}$ is a self-report questionnaire comprising 21 questions measuring the severity of anxiety based on the 
importance of accurately distinguishing anxiety and depression. The BAI deals with major cognitive, emotional, and physical areas $[49,50]$. The level of discomfort in the past week is assessed by applying a 4-point Likert scale ranging from 0 to 3 points to the symptoms indicated by each item; the total score on the BIA indicates the degree of anxiety [49]. Total scores of $22-26$ points indicate an anxious state, $27-31$ a moderately anxious state, and $\geq 32$ points severe anxiety [51]. The BAI was translated into Korean by Kwon [52], and the Cronbach's alpha was $0.93[49,50]$.

\section{EEG Acquisition and Analyses}

For testing, each consenting participant was seated in a comfortable chair in a sound-attenuated EEG room at Soonchunhyang University Seoul Hospital. The EEG was acquired using a NeuroScan SynAmps amplifier (Compumedics USA, E1 Paso, TX, USA) with 64 Ag-AgCl electrodes mounted on a Quik Cap using an extended 10-20 placement scheme. The ground electrode was located on a participant's forehead, and the physically linked reference electrodes were located at both mastoids. The vertical electrooculogram (EOG) was positioned above and below the left eye, and the horizontal EOG was recorded at the lateral canthus of each eye. The impedance was kept below $10 \mathrm{k} \Omega$. All data were processed with a $0.1-70$ $\mathrm{Hz}$ bandpass filter and sampled at $1,000 \mathrm{~Hz}$. All subjects were asked to relax their entire body and sit comfortably without moving during the examination. They were able to look at the targets on the computer monitor screen comfortably without focusing while listening to the sound stimuli.

The recorded EEG data were preprocessed using CURRY 8. Gross artifacts, such as those caused by movements, were removed through visual inspection by a trained person with no prior information regarding the origin of the data. Artifacts related to eye movement or eye blinks were removed using the mathematical procedure implemented in the preprocessing software. The data were filtered using a $0.1-70 \mathrm{~Hz}$ bandpass filter and epoched from $100 \mathrm{~ms}$ pre-stimulus to $600 \mathrm{~ms}$ post-stimulus. The epochs were subtracted from the average value of the pre-stimulus interval for baseline correction. If any remaining epochs contained significant physiological artifacts (amplitude exceeding $\pm 75 \mu \mathrm{V}$ ) at any of the 62 electrode sites, they were excluded from further analyses.
Only artifact-free epochs were averaged across trials and participants for event-related potential (ERP) analyses.

\section{LDAEP}

Tones of $1,000 \mathrm{~Hz}$ and $140 \mathrm{~ms}$ duration $(10 \mathrm{~ms}$ increase and $10 \mathrm{~ms}$ fall) were presented through MDRXB950N1 headphones (Sony, Tokyo, Japan) at five intensities: 60, 70, 80, 90, and $100 \mathrm{~dB}$ SPL. Auditory stimuli included 500 stimuli with an inter-stimulus interval randomized between 500 and $900 \mathrm{~ms}$. These stimuli were produced by E-Prime software (Psychology Software Tools, Pittsburgh, PA, USA). Then the N1 peak (most negative peak between 50 and $200 \mathrm{~ms}$ from the stimulus) and the $\mathrm{P} 2$ peak (most positive peak between 150 and $300 \mathrm{~ms}$ from the stimulus) were determined at the $\mathrm{Cz}$ electrode at the five intensities for each participant [53]. The evoked $\mathrm{N} 1 / \mathrm{P} 2$ peak-to-peak amplitudes were calculated in response to the five different auditory stimulus intensities $[54,55]$, and LDAEP was calculated as the slope of the linear regression.

\section{Statistical Analyses}

The Shapiro-Wilk test was performed to determine whether data were normally distributed. First, Analysis of variance (ANOVA) test was used to compare continuous data on demographic and psychological variables among the high-suicidality group, low-suicidality group, and control group, and the Bonferroni post-hoc test was used. Fisher's exact test was used for analyses of categorical data. Next, ANCOVA test was used to compare the maximum amplitudes of LDAEP measured at the Cz electrode among the three groups with age, sex and severity of depression (PHQ-9 and BDI-II score) as covariates. The Bonferroni test was used to control for multiple comparisons of statistically significant results. Finally, 5000 bootstrap resamplings were used to correct for multiple correlations $[56,57]$. Statistical significance was set at $p<0.05$ (two tailed). All statistical analyses were performed using SPSS 26 (IBM Co., Armonk, NY, USA).

\section{RESULTS}

\section{Demographic Characteristics}

Table 1 shows the basic demographic and social characteristics of each group. There were no significant differences in the sex proportions among the three groups ( $p=$ 
Table 1. Demographic characteristics of participants $(n=67)$

\begin{tabular}{|c|c|c|c|c|c|c|}
\hline Variable & Total $(n=67)$ & $\begin{array}{l}\text { Normal (A) } \\
\quad(\mathrm{n}=22)\end{array}$ & $\begin{array}{l}\text { MDD with low } \\
\text { suicidal ideation (B) } \\
\qquad(\mathrm{n}=22)\end{array}$ & $\begin{array}{l}\text { MDD with high } \\
\text { suicidal ideation }(C) \\
\quad(n=23)\end{array}$ & $p$ value & Post-hoc test \\
\hline Age & $34.28 \pm 14.32$ & $31.55 \pm 8.74$ & $42.23 \pm 14.44$ & $29.3 \pm 15.7$ & $0.002^{*}$ & $B>A=C$ \\
\hline Sex & & & & & 0.821 & $A=B=C$ \\
\hline Male & $29(43.28)$ & $11(50)$ & 9 (40.91) & 9 (39.13) & & \\
\hline Education & & & & & $0.004^{* *}$ & $A>B=C$ \\
\hline 1 & $5(7.81)$ & $0(0)$ & $2(9.09)$ & $3(14.29)$ & & \\
\hline 2 & $26(40.62)$ & 4 (19.05) & $9(40.91)$ & $13(61.9)$ & & \\
\hline 3 & $26(40.62)$ & $14(66.67)$ & $9(40.91)$ & 3 (14.29) & & \\
\hline 4 & 7 (10.94) & 3 (14.29) & $2(9.09)$ & $2(9.52)$ & & \\
\hline Occupation & & & & & $0.018^{* * *}$ & $A>B=C$ \\
\hline Yes & $50(74.63)$ & $21(95.45)$ & $13(59.09)$ & $16(69.57)$ & & \\
\hline
\end{tabular}

Values are presented as mean \pm standard deviation for age, and number (\%) for sex, education and occupation.

$p$ value is calculated by ANOVA for age, and Fisher's exact test for sex, education and occupation. Post-hoc test is done by Bonferroni test. Education was classified into four categories: junior high school, with or without graduation (1); high school graduate (2); university graduate (3); and graduate degree (4).

*MDD with high suicidal ideation vs. Healthy control, $p=1.000$; MDD with low suicidal ideation vs. Healthy control, $p=0.030$; MDD with high suicidal ideation vs. MDD with low suicidal ideation, $p=0.006$. ${ }^{*}$ MDD with high suicidal ideation vs. Healthy control, $p=0.002 ;$ MDD with low suicidal ideation vs. Healthy control, $p=0.152$; MDD with high suicidal ideation vs. MDD with low suicidal ideation, $p=0.435$. ***MDD with high suicidal ideation vs. Healthy control, $p=0.143$; MDD with low suicidal ideation vs. Healthy control, $p=0.018$, MDD with high suicidal ideation vs. MDD with low suicidal ideation, $p=1.000$.

Table 2. Psychological characteristics of participants

\begin{tabular}{lcccccc}
\hline Questionnaire & Total $(\mathrm{n}=67)$ & $\begin{array}{c}\text { Normal }(\mathrm{A}) \\
(\mathrm{n}=22)\end{array}$ & $\begin{array}{c}\text { MDD with low } \\
\text { suicidal ideation }(\mathrm{B}) \\
(\mathrm{n}=22)\end{array}$ & $\begin{array}{c}\text { MDD with high } \\
\text { suicidal ideation (C) } \\
(\mathrm{n}=23)\end{array}$ & $p$ value & Post-hoc test \\
\hline PHQ-9 & $11.93 \pm 8.74$ & $1.95 \pm 2.17$ & $14.36 \pm 5.71$ & $19.13 \pm 5.84$ & $<0.001^{*}$ & $\mathrm{C}>\mathrm{B}>\mathrm{A}$ \\
BDI-II & $22.61 \pm 16.58$ & $4.05 \pm 3.27$ & $25.77 \pm 9.48$ & $37.35 \pm 12.14$ & $<0.001$ & $\mathrm{C}>\mathrm{B}>\mathrm{A}$ \\
SSI-BECK & $9.81 \pm 10.71$ & $1.18 \pm 1.87$ & $5.0 \pm 4.49$ & $22.65 \pm 7.18$ & $<0.001$ & $\mathrm{C}>\mathrm{B}>\mathrm{A}$ \\
BAI & $21.03 \pm 16.22$ & $5.68 \pm 7.05$ & $25.36 \pm 14.67$ & $31.76 \pm 12.9$ & $<0.001^{* *}$ & $\mathrm{~B}=\mathrm{C}>\mathrm{A}$ \\
State Anxiety in & $53.72 \pm 16.03$ & $35.86 \pm 9.79$ & $61.36 \pm 8.97$ & $63.48 \pm 11.2$ & $<0.001^{* * *}$ & $\mathrm{~B}=\mathrm{C}>\mathrm{A}$ \\
$\quad$ & & & & & & \\
\hline STAI (SAI) & & & & & & \\
\hline
\end{tabular}

Values are presented as mean \pm standard deviation for PHQ-9, BDI-II, SSI-BECK, BAI and SAI.

PHQ-9, Patient Health Questionnaire-9; BDI-II, Beck Depression Inventory-II; SSI, Scale for Suicidal Ideation; BAI, Beck Anxiety Inventory; STAI, State-Trait Anxiety Inventory.

$p$ value is calculated by ANOVA, and post-hoc test is done by Bonferroni test.

*MDD with high suicidal ideation vs. Healthy control, $p<0.001$; MDD with low suicidal ideation vs. Healthy control; $p<0.001$, MDD with high suicidal ideation vs. MDD with low suicidal ideation, $p=0.005 .{ }^{* *} \mathrm{MDD}$ with high suicidal ideation vs. Healthy control, $p<0.001$; MDD with low suicidal ideation vs. Healthy control, $p<0.001$; MDD with high suicidal ideation vs. MDD with low suicidal ideation, $p=0.264$. ${ }^{* * *}$ MDD with high suicidal ideation vs. Healthy control, $p<0.001$; MDD with low suicidal ideation vs. Healthy control, $p<0.001$; MDD with high suicidal ideation vs. MDD with low suicidal ideation, $p=1.000$.

0.821). The healthy control group showed a significantly higher educational background ( $p=0.004$ ) and occupational status ( $p=0.018$ ) compared to the others. The mean age was significantly higher in the low-suicidality group than in the others $(p=0.002)$.

\section{Psychological Characteristics}

Table 2 shows the psychological characteristics of each group. Significant differences among the three groups were observed in the PHQ-9, BDI-II, SSI-BECK, BAI, and SAI (all $p<0.001$ ). The high-suicidality group had significantly higher PHQ-9 ( $p=0.005)$, BDI-II $(p<0.001)$ and SSI-BECK $(p<0.001)$ scores compared to the lowsuicidality group (Table 2 ). BAI and SAI scores were significantly lower for the healthy control group than for the other two groups ( $p<0.001$ ); there were no significant $\mathrm{BAI}$ and SAI differences between the high- and low-suicidality groups. 
A

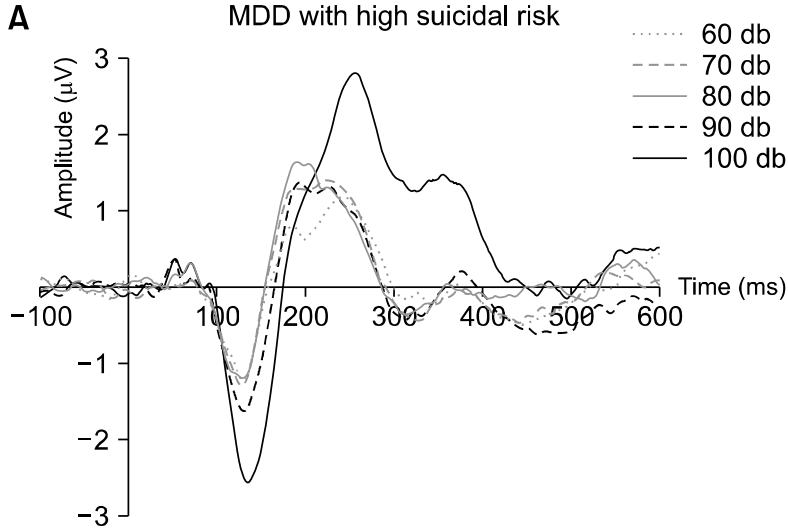

C

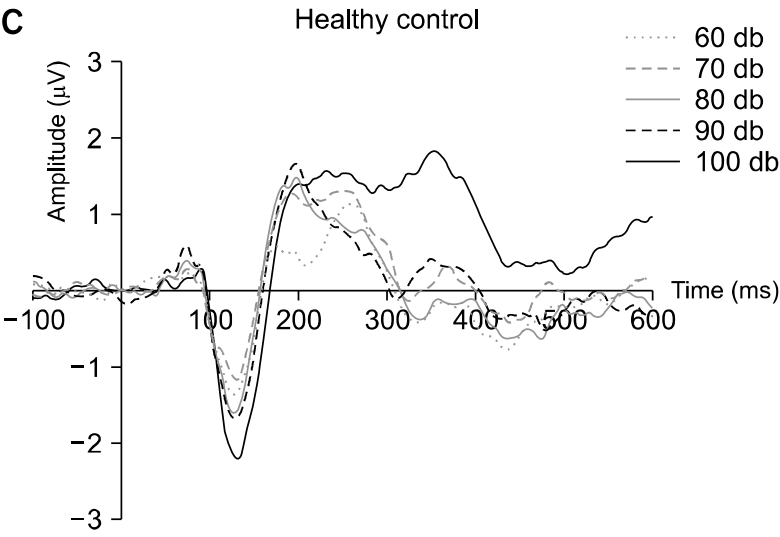

\section{LDAEP}

Average ERPs at the Cz electrode of the LDAEP for each $\mathrm{dB}$ are shown in Figure 1. Statistically significant differences in LDAEP levels were observed among the three groups ( $p=0.007$ ) (Fig. 2). The high-suicidality group had higher LDAEP than the other two groups (high-suicidality group: $0.82 \pm 0.79$; low-suicidality group: $0.26 \pm 0.36$; healthy control group: $0.49 \pm 0.46)$. When the $p$ value was adjusted for each comparison using the Bonferroni test, the high-suicidality group showed a significantly higher LDAEP level than the low-suicidality group ( $p=$ 0.014). However, there were no significant differences in LDAEP levels between the high-suicidality and healthy control groups ( $p=0.281$ ) or between the low-suicidality and healthy control groups $(p=0.236)$.

\section{DISCUSSION}

This cross-sectional study investigated the feasibility of using LDAEP as a candidate for a biomarker for identifying suicide risk. Participants were divided into three groups by level of suicidal ideation, and LDAEP was

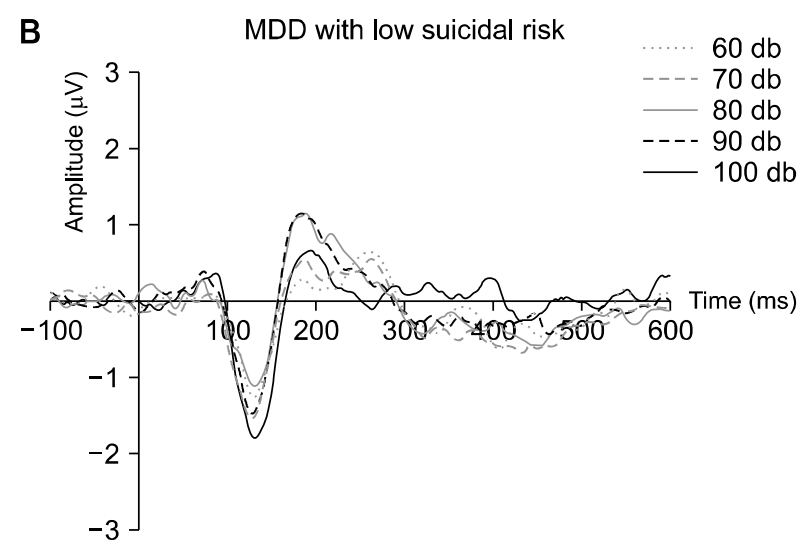

Fig. 1. Grand average of loudness dependence of the auditory evoked potential event-related potentials at the $\mathrm{Cz}$ electrode for MDD patients with high suicidal risk (A), MDD patients with low suicidal risk (B), and healthy controls (C).

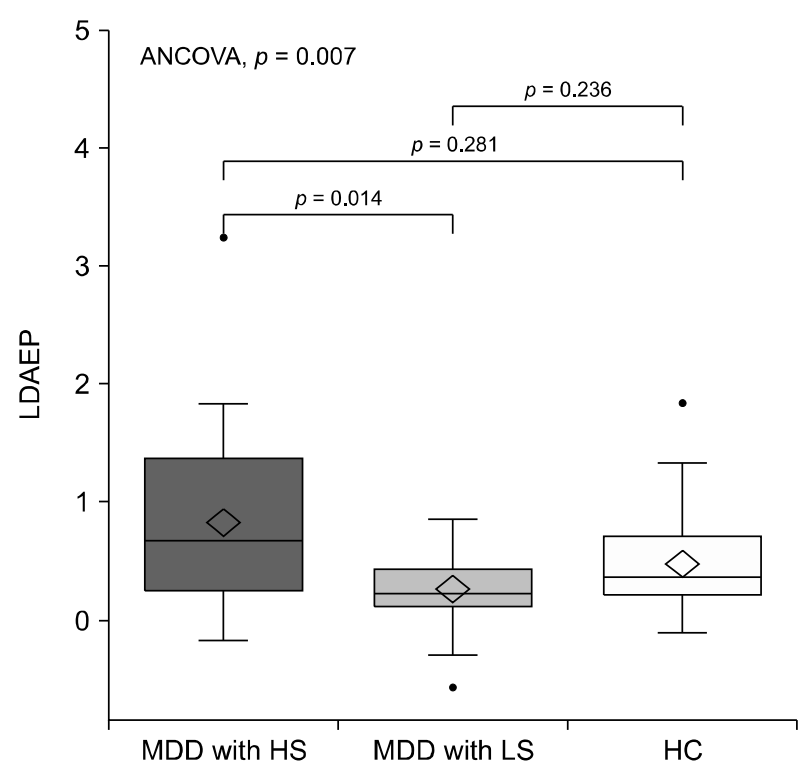

Fig. 2. Box plot of loudness dependence of the auditory evoked potential (LDAEP) event-related potentials at the Cz electrode for MDD patients with high-suicidality (MDD with HS), MDD patients with low-suicidality (MDD with LS), and healthy controls (HC). LDAEP between MDD patients with high-suicidality and MDD patients with low-suicidality shows significant difference $(p=0.014)$. 
measured in each subject. Our results identified some key findings. First, the LDAEP level was higher in the high-suicidality than in the low-suicidality group. Second, there were no significant differences in LDAEP levels between the healthy control group and the overall depression group.

First, LDAEP was higher in the major depressive disorder group with high suicidal ideation than in the major depressive disorder group with low suicidal ideation. Serotonin, a neurotransmitter that is mainly involved in emotional regulation, is widespread in the central nervous system [58]. Abnormalities in serotonin system, such as low presynaptic 5-HT activity, are thought to be associated with dysfunction of regulating mood, impulsive aggressive behavior and suicidal behavior $[59,60]$. Thus, it is hypothesized that serotonergic abnormality makes vulnerability for suicide [61], such as cognitive control of mood, impulsive and aggressive behavior, with noradrenergic and hypothalamic-pituitary-adrenal axis stressresponse system impairment [62]. The 5-HT Hypothesis proposed by Mann et al. [63] is related to suicide and central serotonin. In the basis of this hypothesis, a post-mortem study of suicide attempters found that the concentrations of 5-HT and 5-hydroxyindoleacetic acid (5-HIAA) in brain tissue were lower than those in healthy people [63]. The 5-HT receptors were increased and the serotonin receptor binding sites decreased in the prefrontal cortex after suicide attempts [64]. In conclusion, Mann et al. [63] suggested that serotonin is associated with suicidality, including suicidal ideation and suicidal attempt. Based on the 5-HT hypothesis, we can conclude that measuring serotonin activity in the central system may be meaningful for identifying the risk for suicidal ideation or suicide attempts.

Following Mann's study, numerous other studies confirmed the relationship between suicidal ideation or a history of suicide attempt and serotonin [32,65-67]. In aspects of suicidal ideation, Patients with confirmed acute suicidal ideation on item 3 of the Hamilton Depression Scale (HAMD) were found to have higher LDAEP than those without suicidal ideation [33]. A negative correlation was found between suicidal ideation and LDAEP in patients with atypical depression [68]. One study [24] revealed since SSI-BECK score was significantly different from suicidal attempters and non-suicidal attempters, strong LDAEP may be related to an acute suicidal idea. In aspects of a history of suicidal attempt, 5-HT and 5-HIAA, a serotonin metabolite, were reduced in cerebrospinal fluid in patients with suicide attempts [69]. The number of 5 -HT2 receptors in the frontal lobe was increased in suicide attempters [70]. In addition, the attention and executive functions associated with the frontal lobe were reduced in depressive patients with suicide attempts [71]. Also levels of LDAEP, a serotonin activity indicator, were higher in patients with a history of suicide attempts [24,31]. In aspects of acute suicidal attempt, increased LDAEP was found 9 days after suicidal attempt, while LDAEP was low in 2, 5 and 16 days after attempt [72]. Other study revealed LDAEP was strongest 5 days after suicidal attempt or suicidal behavior [32]. Nevertheless, controversy over the relationship between suicide and LDAEP persists. To the best of our knowledge, studies that found no significant association either used small sample or were not designed to reveal the association between LDAEP and suicide $[73,74]$.

In the present study, LDAEP was used to find the association between suicide and serotonin in patients with major depression. The results of the present study could be used as a basis for the hypothesis that higher LDAEP values in patients with major depressive disorder suggest a higher risk for suicide. However, one study [33] reported that LDAEP was lower in patients with a history of attempted suicide in various psychiatric disorders such as alcoholism, depression, and bipolar disorder than in a control group, but was high in depressive patients with acute suicidal ideation. This means that changes in the serotonin system related to suicidal ideation or behavior are not constant and can change depending on the type of disease or the acute severity of suicidal ideation. Therefore, whether LDAEP can be used as a suicide risk indicator in cases of major depression accompanied by other diseases that affect suicide, such as personality disorder and anxiety disorder, is unclear. In addition, the relationship between LDAEP and suicidal ideation or suicidal behavior in psychiatric disorders other than major depression requires further research. In future studies, it will be important to distinguish between current suicidal ideation and past history of suicide attempts.

Second, there were no significant differences in LDAEP between the normal control group and the major depressive group regardless of the severity of suicidal ideation, consistent with previous reports $[30,32,75]$. Dysfunctions 
in serotonin transmitters and $5-\mathrm{HT}_{1 \mathrm{~A}}$ receptors are thought to be the primary etiologies in major depression $[76,77]$, and serotonin levels of depressive patients, measured in the platelets, were significantly lower than those in the normal group [78]. Thus, we suggest that serotonin and major depression are related. In addition, we must consider that selective serotonin receptor blockers, which modulate serotonin receptors, are used as a treatment agent for major depression based on these points [79]. With regard to the finding that serotonin and major depression could be related, there may still be controversy as to whether LDAEP has any relationship with major depression. However, considering the results of the present study and related studies, we suggest that it will be difficult to use LDAEP as a consistent state marker in major depression. In our study, the result showing no significant difference between the healthy control group and the depression group may be explained in two ways. First, LDAEP may reflect other symptoms affected by the serotonin system, such as decreased attention and impulsiveness, in addition to depression [80]. Second, major depression may be due to complex actions of neurotransmitter systems such as norepinephrine, as well as to other pre- and post-synaptic receptors and gene regulation $[81,82]$.

There are some limitations to the interpretation of these results. First, it is difficult to generalize our results due to the small sample size. It is necessary to recruit a large number of subjects to conduct research on the relationship between LDAEP and suicidal ideation in future studies. Second, the mean age was higher in the low-suicidality group than in the high-suicidality group. It is known that aging could affect central serotonergic activity and serotonin receptor activity $[83,84]$. Thus, in our study, age is analyzed as covariant in comparing the difference of LDAEP between the groups by ANCOVA. Another study [73] showed that the effects of age on serotonin differ by sex. Thus, further studies on the relationships among serotonin activity, sex, and age are needed in the future. Third, we did not conduct our study according to the timing of suicidal ideation or of suicide attempts. Uhl et al. [72] reported that LDAEP was highest around 9 days after suicide attempts, suggesting that suicidal ideation and attempts may not cause an immediate change in LDAEP, and the effects on serotonin activity may be delayed by about a week. In addition, Juckel and Hegerl [33] reported that LDAEP levels are high in patients with acute suicidal ideations, but patients with a history of suicide attempts have low LDAEP levels. Future studies should consider the time when suicidal ideation occurred. Fourth, the study was conducted without distinguishing smokers from non-smokers. Generally, smokers have lower levels of LDAEP than non-smokers [85], and smokers with major depression are also known to have lower LDAEP levels than non-smokers with major depression [73]. This is probably because smoking is related to the gene involved in 5-HT regulation [73]. Meanwhile, a previous study showed that smoking itself lowers serotonin activity and increases the risk for suicide attempts [86]. Future studies need to clarify the link between smoking and LDAEP.

\section{Implications}

Currently, suicide is increasingly common worldwide, and researchers in mental health fields are working to find biomarkers for early detection of suicide risk. We suggest that LDAEP is a less invasive and economical tool for measuring serotonin activity related to suicidal ideation and behavior, in addition to being as a candidate for a biomarker of suicide risk in patients with major depression. Our results extend those of previous studies and contribute to the identification of suicidality in depressed patients by LDAEP. Future research should examine the usefulness of LDAEP as a reliable tool for early prevention of suicide.

\section{- Acknowledgments}

This work was supported by the Korean Neuro Psychiatric Association (KNPA) Jisan Lee Sejong Fund. The funders had no role in study design, data collection and analysis, the decision to publish, or preparation of the manuscript.

\section{Conflicts of Interest}

No potential conflict of interest relevant to this article was reported.

\section{Author Contributions}

Conceptualization: Yeon Jung Lee. Data acquisition: Mingyu Hwang, Yeon Jung Lee, Minji Lee, Byungjoo Kang, Yun Sung Lee, Jaeuk Hwang, Sung-il Woo, and Sang-Woo Hahn. Formal analysis: Mingyu Hwang and Yeon Jung Lee. Funding: Korean Neuropsychiatric association Jisan Lee Sejong Fund, Soonchunhyang University Research Fund. Supervision: Sang-Woo Hahn. Writing- 
original draft: Mingyu Hwang. Writing - review \& editing: Mingyu Hwang, Yeon Jung Lee, and Sang-Woo Hahn.

\section{ORCID}

Mingyu Hwang https://orcid.org/0000-0002-5805-2335

Yeon Jung Lee https://orcid.org/0000-0001-8953-5893

Minji Lee Byungjoo Kang Yun Sung Lee https://orcid.org/0000-0003-4962-3869 https://orcid.org/0000-0001-5182-9730 Jaeuk Hwang Sung-il Woo Sang-Woo Hahn https://orcid.org/0000-0002-5110-7225 https://orcid.org/0000-0003-0528-3305 https://orcid.org/0000-0002-1661-095X https://orcid.org/0000-0003-1662-5438

\section{REFERENCES}

1. K.indicator. The suicide rate in Korea [Internet]. Daejeon: Statistics Korea; 2018 [cited at $2020 \mathrm{Jul} \mathrm{1].} \mathrm{Available} \mathrm{from:}$ http://www.index.go.kr/unify/idx-info.do?idxCd=8040.

2. Hyun KR, Lee SM. Socio-economic cost analysis of the top five causes of death in Korea. Health Insur Policy 2014;13: 91-107.

3. Klonsky ED, May A. Rethinking impulsivity in suicide. Suicide Life Threat Behav 2010;40:612-619.

4. Pokorny AD. Prediction of suicide in psychiatric patients. Report of a prospective study. Arch Gen Psychiatry 1983;40: 249-257.

5. Kim J, Kim M, Kim YR, Cho KH, Lee KU. High prevalence of psychotropics overdose among suicide attempters in Korea. Clin Psychopharmacol Neurosci 2015;13;302-307.

6. Lee Y, Lee D, Hong HJ. Gender-based multilevel analysis of influential factors for suicide attempts among at-risk non-referred adolescents in Korea. Clin Psychopharmacol Neurosci 2020;18;116-126.

7. Chamberlain P, Goldney R, Delfabbro P, Gill T, Dal Grande L. Suicidal ideation. The clinical utility of the K10. Crisis 2009; 30:39-42.

8. Bryan CJ, Rudd MD. Advances in the assessment of suicide risk. J Clin Psychol 2006;62:185-200.

9. Chang BP, Franklin JC, Ribeiro JD, Fox KR, Bentley KH, Kleiman EM, et al. Biological risk factors for suicidal behaviors: a meta-analysis. Transl Psychiatry 2016;6:e887.

10. Corrigan MH, Gillette GM, Quade D, Garbutt JC. Panic, suicide, and agitation: independent correlates of the TSH response to TRH in depression. Biol Psychiatry 1992;31:984992.

11. Sudol K, Mann JJ. Biomarkers of suicide attempt behavior: towards a biological model of risk. Curr Psychiatry Rep 2017; 19:31.

12. Sullivan GM, Mann JJ, Oquendo MA, Lo ES, Cooper TB, Gorman JM. Low cerebrospinal fluid transthyretin levels in depression: correlations with suicidal ideation and low serotonin function. Biol Psychiatry 2006;60:500-506.
13. Krakowski M. Violence and serotonin: influence of impulse control, affect regulation, and social functioning. J Neuropsychiatry Clin Neurosci 2003;15:294-305.

14. Oades RD. Role of the serotonin system in ADHD: treatment implications. Expert Rev Neurother 2007;7:1357-1374.

15. Underwood MD, Kassir SA, Bakalian MJ, Galfalvy H, Dwork AJ, Mann JJ, et al. Serotonin receptors and suicide, major depression, alcohol use disorder and reported early life adversity. Transl Psychiatry 2018;8:279.

16. Bondy B, Kuznik J, Baghai T, Schüle C, Zwanzger P, Minov C, et al. Lack of association of serotonin-2A receptor gene polymorphism (T102C) with suicidal ideation and suicide. Am J Med Genet 2000;96:831-835.

17. Du L, Bakish D, Lapierre YD, Ravindran AV, Hrdina PD. Association of polymorphism of serotonin $2 A$ receptor gene with suicidal ideation in major depressive disorder. Am JMed Genet 2000;96:56-60.

18. Höfer P, Schosser A, Calati R, Serretti A, Massat I, Kocabas NA, et al. The impact of serotonin receptor $1 A$ and $2 A$ gene polymorphisms and interactions on suicide attempt and suicide risk in depressed patients with insufficient response to treatment--a European multicentre study. Int Clin Psychopharmacol 2016;31:1-7.

19. Ryding E, Lindström M, Träskman-Bendz L. The role of dopamine and serotonin in suicidal behaviour and aggression. Prog Brain Res 2008; 172:307-315.

20. Turecki G, Brent DA. Suicide and suicidal behaviour. Lancet 2016;387:1227-1239.

21. Oquendo MA, Placidi GP, Malone KM, Campbell C, Keilp J, Brodsky B, et al. Positron emission tomography of regional brain metabolic responses to a serotonergic challenge and lethality of suicide attempts in major depression. Arch Gen Psychiatry 2003;60:14-22.

22. Carley DW, Radulovacki M. Role of peripheral serotonin in the regulation of central sleep apneas in rats. Chest 1999;115: 1397-1401.

23. Coccaro EF. Central serotonin and impulsive aggression. Br J Psychiatry Suppl 1989;(8):52-62.

24. Kim DH, Park YM. The association between suicidality and serotonergic dysfunction in depressed patients. J Affect Disord 2013;148:72-76.

25. Juckel G, Molnár M, Hegerl U, Csépe V, Karmos G. Auditoryevoked potentials as indicator of brain serotonergic activity--first evidence in behaving cats. Biol Psychiatry 1997;41:11811195.

26. Nathan PJ, Segrave R, Phan KL, O’ Neill B, Croft RJ. Direct evidence that acutely enhancing serotonin with the selective serotonin reuptake inhibitor citalopram modulates the loudness dependence of the auditory evoked potential (LDAEP) marker of central serotonin function. Hum Psychopharmacol 2006; 21:47-52.

27. Wutzler A, Winter C, Kitzrow W, Uhl I, Wolf RJ, Heinz A, et al. Loudness dependence of auditory evoked potentials as in- 
dicator of central serotonergic neurotransmission: simultaneous electrophysiological recordings and in vivo microdialysis in the rat primary auditory cortex. Neuropsychopharmacology 2008;33:3176-3181.

28. Hegerl U, Juckel G. Intensity dependence of auditory evoked potentials as an indicator of central serotonergic neurotransmission: a new hypothesis. Biol Psychiatry 1993;33:173187.

29. Gudlowski Y, Ozgürdal S, Witthaus H, Gallinat J, Hauser M, Winter $\mathrm{C}$, et al. Serotonergic dysfunction in the prodromal, first-episode and chronic course of schizophrenia as assessed by the loudness dependence of auditory evoked activity. Schizophr Res 2009;109:141-147.

30. Park YM, Lee SH, Kim S, Bae SM. The loudness dependence of the auditory evoked potential (LDAEP) in schizophrenia, bipolar disorder, major depressive disorder, anxiety disorder, and healthy controls. Prog Neuropsychopharmacol Biol Psychiatry 2010;34:313-316.

31. Chen TJ, Yu YW, Chen MC, Wang SY, Tsai SJ, Lee TW. Serotonin dysfunction and suicide attempts in major depressives: an auditory event-related potential study. Neuropsychobiology 2005;52:28-36.

32. Graßnickel V, Illes F, Juckel G, Uhl I. Loudness dependence of auditory evoked potentials (LDAEP) in clinical monitoring of suicidal patients with major depression in comparison with non-suicidal depressed patients and healthy volunteers: a follow-up-study. J Affect Disord 2015;184:299-304.

33. Juckel G, Hegerl U. Evoked potentials, serotonin, and suicidality. Pharmacopsychiatry 1994;27 Suppl 1:27-29.

34. Brent DA, Perper JA, Goldstein CE, Kolko DJ, Allan MJ, Allman CJ, et al. Risk factors for adolescent suicide. A comparison of adolescent suicide victims with suicidal inpatients. Arch Gen Psychiatry 1988;45:581-588.

35. Pelkonen M, Marttunen M, Pulkkinen E, Laippala P, Aro $\mathrm{H}$. Characteristics of out-patient adolescents with suicidal tendencies. Acta Psychiatr Scand 1997;95:100-107.

36. Holi MM, Pelkonen M, Karlsson L, Kiviruusu O, Ruuttu T, Heilä $\mathrm{H}$, et al. Psychometric properties and clinical utility of the Scale for Suicidal Ideation (SSI) in adolescents. BMC Psychiatry 2005;5:8.

37. First MB. User's guide for the structured clinical interview for DSM-IV-TR axis I disorders : SCID-I. New York:New York State Psychiatric Institute;2002.

38. Burkey JM, Lippy WH, Schuring AG, Rizer FM. Clinical utility of the 512-Hz Rinne tuning fork test. Am J Otol 1998;19:59-62.

39. Beck AT, Steer RA, Ranieri WF. Scale for suicide ideation: psychometric properties of a self-report version. J Clin Psychol 1988:44:499-505.

40. Shin MS, Park KB, Oh KJ, Kim ZS. A study of suicidal ideation among high school students: the structural relation among depression, hopelessness, and suicidal ideation. Korean J Clin Psychol 1990;9:1-19.

41. Kroenke K, Spitzer RL, Williams JB. The PHQ-9: validity of a brief depression severity measure. J Gen Intern Med 2001; 16:606-613.

42. Han C, Jo SA, Kwak JH, Pae CU, Steffens D, Jo I, et al. Validation of the Patient Health Questionnaire-9 Korean version in the elderly population: the Ansan geriatric study. Compr Psychiatry 2008;49:218-223.

43. Park SJ, Choi HR, Choi JH, Kim KW, Hong JP. Reliability and validity of the Korean version of the Patient Health Questionnaire-9 (PHQ-9). Anxiety Mood 2010;6:119-124.

44. Beck AT, Steer RA, Brown GK. BDI-II, Beck depression inventory: manual. San Antonio:Psychological Corp.;1996. p.490-498.

45. Sung H, Kim J, Park Y, Bai D, Lee S, Ahn H. A study on the reliability and the validity of Korean version of the Beck Depression Inventory-II (BDI-II). J Korean Soc Biol Ther Psychiatry 2008;14:201-212.

46. Spielberger CD, Gorsuch RL, Lushene RE. STA/ manual for the state-trait anxiety inventory ("Self-evaluation questionnaire"). Palo Alto:Consulting Psychologists Press;1970. p. 1-24.

47. Spielberger CD. Anxiety: state-trait process. In: Spielberger $C D$, Sarason $I G$, editors. Stress and anxiety. Washington D.C.:Hemisphere Pub.;1975. p.115-143.

48. Kim J, Shin D. A study based on the standardization of the STAl for Korea. New Med J 1978;21:69-75.

49. Beck AT, Epstein N, Brown G, Steer RA. An inventory for measuring clinical anxiety: psychometric properties. J Consult Clin Psychol 1988;56:893-897.

50. Yook SP, Kim ZS. A clinical study on the Korean version of Beck Anxiety Inventory: comparative study of patient and non-patient. Korean J Clin Psychol 1997;16:185-197.

51. Park HJ, Kim HN, Kim IB, Jeon SA. Reliability of the Beck Depression Inventory in adolescence. J Korean Acad Fam Med 2000;21:244-253.

52. Kwon SM, Oei TPS. Differential causal roles of dysfunctional attitudes and automatic thoughts in depression. Cogn Ther Res 1992;16:309-328.

53. Hagenmuller F, Heekeren K, Meier M, Theodoridou A, Walitza S, Haker $\mathrm{H}$, et al. The loudness dependence of auditory evoked potentials (LDAEP) in individuals at risk for developing bipolar disorders and schizophrenia. Clin Neurophysiol 2016;127:1342-1350.

54. Gallinat J, Bottlender R, Juckel G, Munke-Puchner A, Stotz G, Kuss $\mathrm{HJ}$, et al. The loudness dependency of the auditory evoked N1/P2-component as a predictor of the acute SSRI response in depression. Psychopharmacology (Berl) 2000;148: 404-411.

55. Hegerl U, Gallinat J, Juckel G. Event-related potentials. Do they reflect central serotonergic neurotransmission and do they predict clinical response to serotonin agonists? J Affect Disord 2001;62:93-100.

56. Kim JS, Kim DW, Kwon YJ, Lee HY, Kim S, Shim SH. The relationship between auditory evoked potentials and symptoms of attention-deficit/hyperactivity disorder in adult patients 
with major depressive disorder. Int I Psychophysiol 2019; 142:50-56.

57. Pernet CR, Chauveau N, Gaspar C, Rousselet GA. LIMOEEG: a toolbox for hierarchical LInear MOdeling of ElectroEncephaloGraphic data. Comput Intell Neurosci 2011;2011:831409.

58. Lee JH, Kim HT, Hyun DS. Possible association between serotonin transporter promoter region polymorphism and impulsivity in Koreans. Psychiatry Res 2003;118:19-24.

59. Asberg M, Forslund K. Neurobiological aspects of suicidal behaviour. Int Rev Psychiatr 2000;12:62-74.

60. Coccaro EF, Siever LJ, Klar HM, Maurer G, Cochrane K, Cooper TB, et al. Serotonergic studies in patients with affective and personality disorders. Correlates with suicidal and impulsive aggressive behavior. Arch Gen Psychiatry 1989;46: 587-599.

61. Mann JJ. The neurobiology of suicide. Nat Med 1998;4:25-30.

62. van Heeringen K, Mann JJ. The neurobiology of suicide. Lancet Psychiatry 2014;1:63-72.

63. Mann JJ, Arango V, Marzuk PM, Theccanat S, Reis DJ. Evidence for the 5-HT hypothesis of suicide. A review of post-mortem studies. Br J Psychiatry Suppl 1989;(8):7-14.

64. Mann JJ, Huang YY, Underwood MD, Kassir SA, Oppenheim $\mathrm{S}$, Kelly TM, et al. A serotonin transporter gene promoter polymorphism (5-HTTLPR) and prefrontal cortical binding in major depression and suicide. Arch Gen Psychiatry 2000;57:729738.

65. Juckel G, Mavrogiorgou P, Bredemeier S, Gallinat J, Frodl T, Schulz $\mathrm{C}$, et al. Loudness dependence of primary auditorycortex-evoked activity as predictor of therapeutic outcome to prophylactic lithium treatment in affective disorders--a retrospective study. Pharmacopsychiatry 2004;37:46-51.

66. Mann J, Currier D, Stanley B, Oquendo MA, Amsel LV, Ellis SP. Can biological tests assist prediction of suicide in mood disorders? Int J Neuropsychopharmacol 2006;9:465-474.

67. Nordström P, Samuelsson M, Asberg M, Träskman-Bendz L, Aberg-Wistedt A, Nordin C, et al. CSF 5-HIAA predicts suicide risk after attempted suicide. Suicide Life Threat Behav 1994; 24:1-9.

68. Lee SH, Park YC, Yoon S, Kim JI, Hahn SW. Clinical implications of loudness dependence of auditory evoked potentials in patients with atypical depression. Prog Neuropsychopharmacol Biol Psychiatry 2014;54:7-12.

69. Asberg M, Träskman L, Thorén P. 5-HIAA in the cerebrospinal fluid. A biochemical suicide predictor? Arch Gen Psychiatry 1976;33:1193-1197.

70. Mann JJ, Arango V. Integration of neurobiology and psychopathology in a unified model of suicidal behavior. J Clin Psychopharmacol 1992;12(2 Suppl):2S-7S.

71. Keilp JG, Sackeim HA, Brodsky BS, Oquendo MA, Malone KM, Mann JJ. Neuropsychological dysfunction in depressed suicide attempters. Am J Psychiatry 2001;158:735-741.

72. Uhl I, Illes F, Graßnickel V, Echterhoff S, Norra C, Juckel G.
Loudness dependence of auditory evoked potentials (LDAEP) in clinical monitoring of suicidal patients with major depression: a pilot study. Eur Arch Psychiatry Clin Neurosci 2012;262:487-492.

73. Min JA, Lee SH, Lee SY, Chae JH, Lee CU, Park YM, et al. Clinical characteristics associated with different strengths of loudness dependence of auditory evoked potentials (LDAEP) in major depressive disorder. Psychiatry Res 2012;200:374381.

74. Park YM, Lee BH, Lee SH. The association between serum lipid levels, suicide ideation, and central serotonergic activity in patients with major depressive disorder. J Affect Disord 2014; 159:62-65.

75. Linka T, Sartory G, Bender S, Gastpar M, Müller BW. The intensity dependence of auditory ERP components in unmedicated patients with major depression and healthy controls. An analysis of group differences. J Affect Disord 2007;103:139145.

76. Domínguez-López S, Howell R, Gobbi G. Characterization of serotonin neurotransmission in knockout mice: implications for major depression. Rev Neurosci 2012;23:429-443.

77. Savitz J, Lucki I, Drevets WC. 5-HT(1A) receptor function in major depressive disorder. Prog Neurobiol 2009;88:17-31.

78. Le Quan-Bui KH, Plaisant O, Leboyer M, Gay C, Kamal L, Devynck MA, et al. Reduced platelet serotonin in depression. Psychiatry Res 1984;13:129-139.

79. Mann JJ. Role of the serotonergic system in the pathogenesis of major depression and suicidal behavior. Neuropsychopharmacology 1999;21(2 Suppl):99S-105S.

80. Banerjee E, Nandagopal K. Does serotonin deficit mediate susceptibility to ADHD? Neurochem Int 2015;82:52-68.

81. Alda M. Genetic factors and treatment of mood disorders. Bipolar Disord 2001;3:318-324.

82. Nutt DJ. Relationship of neurotransmitters to the symptoms of major depressive disorder. J Clin Psychiatry 2008;69 Supp/ E1:4-7.

83. Cidis Meltzer C, Drevets WC, Price JC, Mathis CA, Lopresti B, Greer PJ, et al. Gender-specific aging effects on the serotonin 1A receptor. Brain Res 2001;895:9-17.

84. Pogarell O, Tatsch K, Juckel G, Hamann C, Mulert C, Pöpperl $\mathrm{G}$, et al. Serotonin and dopamine transporter availabilities correlate with the loudness dependence of auditory evoked potentials in patients with obsessive-compulsive disorder. Neuropsychopharmacology 2004;29:1910-1917.

85. Gallinat J, Kunz D, Lang UE, Kalus P, Juckel G, Eggers J, et al. Serotonergic effects of smoking are independent from the human serotonin transporter gene promoter polymorphism: evidence from auditory cortical stimulus processing. Pharmacopsychiatry 2005;38:158-160.

86. Malone KM, Waternaux C, Haas GL, Cooper TB, Li S, Mann JJ. Cigarette smoking, suicidal behavior, and serotonin function in major psychiatric disorders. Am J Psychiatry 2003;160: 773-779. 